\title{
La sanità tra conservazione e necessità di cambiamento. EBM e cambiamenti epidemiologici
}

\author{
Antonio Barracca \\ Medico, specialista in Nefrologia e Urologia, esperto di applicazioni per medici su piattaforma Android e Apple, Cagliari
}

The HeALTH CARE SYSTEM BETWEen TRADITION AND NEED OF CHANGE

Abstract. During the last years we have been facing several changes to which we were not prepared, despite elsewhere these changes had already been addressed. Health care costs are becoming progressively higher and somewhat unsustainable due to demographic changes that are leading to a gradual aging of our population thanks to the low birth rates and longer life expectancy. Very often, however, longer life is lived in solitude. Therefore, the larger resources invested do not match the desired health outcomes.

The aging of the population, the general well-being that has eradicated many diseases, and a sedentary lifestyle that has determined other conditions, all have increased degenerative and cardiovascular diseases, as well as tumors, along with the increasing incidence of obesity and diabetes. Therefore, more organs are simultaneously affected for the same reasons and by the same risk factors. Thus, we need to start aggregating the many fragmented medical cultures to put the human being at the centre of the health care system.

Therefore, we should recognize that the organizational models must adapt to the epidemiological changes. These adaptations involve different professions, doctors, and nurses that need to change their role, power, and responsibilities, as well as the approach to their work. This requires them to work not as individual actors, but as protagonists in the care of sick patients, with multiple organs affected, and no longer as purely treating the diseased organs.

Key words: Organizational models, Medical skills, Assessments, Career progression

Conflict of interest: None.

Financial support: None.

Accettato: 26 Novembre 2014

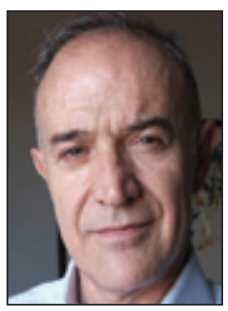

Antonio Barracca

\section{La sfida del cambiamento}

In questi anni, si stanno affrontando sfide alle quali non eravamo preparati, anche se, di queste tematiche, di queste sfide, in altre parti del mondo si discute da anni.

I costi dell'assistenza sanitaria stanno diventando progressivamente più alti $\mathrm{e}$, per certi versi, insostenibili. Le cause di tutto ciò sono diverse.

Stiamo assistendo a profondi cambiamenti demografici che stanno portando a un progressivo invecchiamento delle nostre popolazioni a cui concorrono la bassa natalità e l'allungamento delle aspettative di vita. La vita più lunga, però, spesso è accompagnata da una sua cattiva qualità per la presenza di malattie degenerative e dei tumori. Sempre più spesso, inoltre, la vita più lunga è vissuta e affrontata in solitudine.

A tutto ciò, tuttavia, non corrisponde un miglioramento dei risultati intesi come guarigione e miglioramento della qualità della vita, perché si sta imponendo un modello di medicina basato prevalentemente sulle cure mediche delle malattie che si sono già manifestate nella loro gravità. A maggiori risorse investite, quindi, non corrispondono i risultati di salute auspicati. Non bisogna onestamente, però, ignorare che, negli anni, questo quadro migliorerà, se la ricerca scientifica darà i suoi frutti nel campo soprattutto della medicina rigenerativa (essa però non è alle porte e non sarà alla portata di tutti).

Da queste considerazioni, è nata la filosofia dell'Evidence Based Medicine (EBM), della Medicina Basata sulle Evidenze, rivolta a promuovere, attraverso la sistematica revisione delle evidenze scientifiche disponibili, l'utilizzo di quelle procedure di diagnosi, prognosi e terapia per le quali esistano prove inoppugnabili di efficacia. L'EBM è il processo di ricerca sistematica, di apprendimento e, quindi, di utilizzo dei risultati della ricerca clinica come base per le decisioni cliniche. Il termine EBM fu coniato alla McMaster Medical School in Canada nel 1980 e, con esso, si voleva superare 
il gap da sempre esistente fra i risultati della ricerca e la pratica clinica. I medici, infatti, tendono a prendere decisioni cliniche spesso senza disporre di informazioni sufficienti e di buona qualità. Quindi, i medici e le società scientifiche stanno tentando di fare la propria parte per poter dare le cure più appropriate, quelle con il migliore rapporto costo-beneficio, alla comunità.

Ma altri cambiamenti epidemiologici hanno mutato una realtà clinica che si riteneva immutabile.

Agli inizi degli anni '80, la clinica dei pazienti ricoverati in ospedale era profondamente diversa da quella che vediamo oggi, perché non erano ancora evidenti, nel nostro paese, i cambiamenti che avrebbero segnato i profondi mutamenti epidemiologici della società dei nostri anni. L'aspettativa di vita della popolazione non raggiungeva i valori attuali e le malattie si verificavano in persone relativamente giovani, per cui le corsie degli ospedali erano popolate in prevalenza da persone spesso nel pieno delle loro forze. Ma l'aspetto più interessante era che i pazienti erano ammalati di una sola malattia o a un solo organo. E la malattia, ovviamente, iniziava nel cuore, nel fegato, nei reni, nei polmoni e nel cervello che erano, perciò, gli organi e, quindi, la malattia da curare.

In questi ultimi 30 anni, però, tutto sta nuovamente cambiando. Abbiamo capito che l'invecchiamento della popolazione, il benessere che ha cancellato molte malattie e la sedentarietà che ne ha proposto di nuove hanno fatto esplodere non solo le malattie degenerative, ma le malattie cardiovascolari e i tumori, con il collante dell'obesità e del diabete. Per cui, più organi si ammalano, contemporaneamente, per le stesse cause e per gli stessi fattori di rischio. Diabete, ipertensione, obesità e alimentazione possono essere la causa di malattie che interessano il cuore, i reni e il cervello, contemporaneamente. Quindi, non dobbiamo più curare un organo ammalato, ma un uomo ammalato a più organi. Perciò bisogna ricominciare ad aggregare le Divisioni e accorpare culture mediche per rimettere l'uomo al centro delle cure.

Pertanto, è diventato molto arduo pensare che vi sia, in questi pazienti, una malattia prevalente, se queste patologie sono strettamente correlate. Ecco perché è necessario guardare a un Ospedale orientato a valutare la gravità clinica e a commisurare l'intensità delle cure ai bisogni del paziente.

Dobbiamo prendere atto del fatto che i modelli organizzativi devono adeguarsi ai mutati cambiamenti epidemiologici e che questi cambiamenti coinvolgono le figure professionali, medici e infermieri, modificandone il ruolo, le competenze e le responsabilità e il modo di lavorare. Essi non dovranno più occuparsi solamente della cura degli organi ammalati, come hanno sempre fatto, ma sempre più dovranno prendersi cura di pazienti ammalati a più organi.

Non è, perciò, superfluo ribadire che al servizio sanitario si deve accedere per concorso e per competenze.

Concorsi e competenze devono valere per tutte le funzioni, perché la collettività deve essere certa che le risorse impiegate vengano spese con un rapporto costo-beneficio ottimale.

Negli anni, questo vincolo si è progressivamente affievolito, per cui, per accedere ad alcuni servizi particolarmente impegnativi dal punto di vista delle competenze professionali, si sono create delle graduatorie basate esclusivamente sull'anzianità di servi- zio. Da queste graduatorie si è spesso, in maniera proditoria, attinto per ricoprire incarichi in strutture ospedaliere. Quindi, di queste figure professionali, che sicuramente si impegnano tanto nel loro lavoro, ma che non hanno superato nessun esame e nessuna prova, non conosciamo quale sia la vera preparazione. Siamo certi, comunque, che, nel corso dei lunghi anni di carriera, nessuno si porrà il problema di valutarne le capacità.

\section{Ospedali}

Modelli organizzativi, competenze mediche, valutazioni, progressione di carriera.

\section{Dirigente medico}

Come selezionare i dirigenti

Quale livello di preparazione

Quale livello di responsabilità

Aggiornamento per progetti clinici

La complessità della medicina moderna non ci consente più di assumere medici poco esperti e dei quali non conosciamo la capacità di affrontare i problemi complessi che si troveranno di fronte nel loro lavoro. Non si può immaginare che la loro esperienza e le loro competenze pian piano si formino negli anni. A noi serve che si completino, non che si formino. Le scuole di specializzazione troppo spesso non adempiono totalmente a questa funzione. Noi vorremmo che il medico specialista, assunto in un reparto ospedaliero, sia capace dal primo giorno di esprimere una grande autonomia diagnostica e terapeutica. Da subito, perché i cittadini, gli ammalati, devono sapere che cosa il medico che hanno di fronte sa fare e quello che non sa fare e che non deve fare. Facciamo un esempio, ma è valido per tutte le ipotesi. Un reparto di emodinamica ha bisogno di assumere uno specialista in cardiologia perché deve aumentare il numero degli interventi di emodinamica. Non può pensare di assumere uno specialista in cardiologia che non ha mai fatto un'angioplastica.

Gli ospedali devono assumere medici con le competenze richieste già acquisite.

Abbiamo, perciò, bisogno di un ospedale di insegnamento, di un Teaching Hospital, come nelle migliori tradizioni della medicina di lingua inglese e del nord Europa.

Ciò presuppone che gli ospedali rendano note periodicamente le figure mediche necessarie, in modo tale che i medici che ambiscono a quella specialità frequentino per il tempo necessario un istituto nel quale hanno la possibilità di cimentarsi con queste pratiche, di sviluppare una casistica importante e di avere la certificazione delle loro capacità acquisite. Il possesso di queste capacità, una cultura medica generale e competenze informatiche e statistiche dovranno essere titoli di merito indispensabili per l'accesso alla funzione ospedaliera di dirigente medico. Le cose che dovranno contare, avere un peso, non saranno più solo la laurea e la specializzazione, ma le reali capacità cliniche e la voglia di inserirsi e lavorare in gruppo e di guadagnarsi con il tempo e con l'impegno un ruolo dentro le strutture.

Nel tempo, però, è successo che modelli organizzativi in cui erano presenti figure di responsabilità graduate (aiuti e assisten- 
ti) sono stati stravolti (non voglio dare valutazioni di merito) da un'idea di egualitarismo senza alcuna valutazione del merito. Il fatto che i medici ospedalieri siano tutti diventati "dirigenti medici" ha livellato e reso impossibile o molto difficile valutare le competenze del singolo medico. Esso è spesso scomparso e, al suo posto, esiste l'entità del reparto nel quale l'unica figura riconosciuta è solamente quella del Primario. Ma anche la medicina praticata dal singolo medico scompare se essa è diventata medicina di gruppo, in cui nessuno ha responsabilità definite e tutte le decisioni, non solo quelle più importanti, vengono prese sotto la supervisione del Primario. Quindi tutti uguali, stesso stipendio legato all'anzianità di servizio, stesso lavoro, stesso numero di guardie del reparto, stessi diritti. Cioè un sistema ideale nel quale le aspirazioni di carriera scompaiono, non hanno più significato.

Allora, bisogna ripensare a questo modello organizzativo superato, che ha distrutto il rapporto medico-paziente, che non fa distinzione fra chi lavora e chi non fa nulla, fra chi studia, si aggiorna, raccoglie dati clinici e li confronta con casistiche più ampie e chi vive di rendita, fra chi investe le proprie risorse per dotarsi di strumenti che migliorino la sua preparazione e chi aspetta che la ASL gli faccia un corso ECM per godere di una giornata retribuita.

In un mondo come quello della medicina che cambia a velocità sostenuta, bisogna ripensare alla valutazione periodica della preparazione medica e infermieristica, ogni cinque anni, con una valutazione severa da parte di una commissione esterna, come nei paesi anglosassoni, o con altri mezzi, ma comunque vincolando la progressione della carriera e parte della retribuzione alle competenze e alla valutazione del merito.

E, quindi, sarebbe opportuno ridisegnare il modello organizzativo sulla base della gravità clinica dei pazienti. In un programma che dovrà essere impostato per trasformare i nostri ospedali per acuti in ospedali a intensità di cura, programma che andrà attentamente inserito in questo contesto ostile ai cambiamenti, sclerotizzato e poco pronto al cambiamento e al confronto con altre realtà, sarà cosa saggia iniziare a creare, cominciando dai reparti di chirurgia e medicina generali, 2-4 posti letto dedicati ai pazienti che hanno bisogno di cure intensive. Ciò per interrompere quella insana abitudine di mettere insieme pazienti complessi e in condizioni critiche e pazienti che necessitano di una bassa intensità di cure. Già questo provvedimento, accompagnato dalla scelta di medici e infermieri che si occupino solo di questi pazienti, metterà le basi per una nuova medicina. E poi bisogna liberare le risorse umane troppo a lungo compresse. E noto che i medici, per le nostre leggi, hanno completa autonomia clinica. Bisogna realizzarla di fatto. Quindi, i medici che si occupano di clinica o di chirurgia dovranno avere la responsabilità diretta della diagnosi, delle terapie anche chirurgiche e della prognosi dei pazienti affidati a loro. Questo è l'unico modo per far crescere la responsabilità individuale, per valutare le capacità organizzative e cliniche, per rinsaldare il rapporto fra medico curante e paziente e, infine, per poter fare una valutazione che sia basata sul merito. Ovviamente, dietro tutto ciò, dovrà esserci il coordinamento del Direttore di struttura perché insieme si mettano in pratica Linee Guida condivise, basate sulle evidenze disponibili e sostenute dall'esperienza e dalla casistica maturata nelle strutture.

\section{Direttore di struttura semplice}

Negli ospedali moderni, le strutture semplici hanno assunto un ruolo centrale legato sia all'intensità delle cure a cui si è accennato sia al fatto che, negli anni, le aumentate conoscenze scientifiche hanno portato a enfatizzare aspetti legati tra loro della medicina. Sono sorte la medicina dell'area critica (critical care medicine) e la nefrologia dell'area critica (critical care nephrology). Tutti settori in forte trasformazione culturale e organizzativa che hanno bisogno di una loro più specifica identità e che sono riusciti a mettere insieme competenze diverse al letto del paziente.

La domanda che ci dobbiamo porre è: quale livello dobbiamo stabilire nell'assegnare la responsabilità di queste strutture? Esistono, ovviamente, delle norme di riferimento, ma ogni amministrazione dovrebbe puntare a una selezione per concorso che valuti curriculum scientifico, pratica clinica e capacità organizzativa, dando pochissimo peso all'anzianità.

Questi incarichi non possono diventare fonte di privilegi, ma sono strettamente connessi a progressione di carriera, a regressione se il caso e, comunque sia, a un'assunzione di responsabilità completa.

\section{Direttore di struttura complessa}

Dalla riforma Bindi e quindi con la trasformazione in Aziende delle Unità Sanitarie Locali le Divisioni Mediche e Chirurgiche hanno preso il nome di Strutture Complesse. Anche il responsabile che è sempre stato impersonificato nel "Primo", nel Primario, nel più bravo, nel più competente ha dovuto cambiare nome. La dizione di Direttore di struttura complessa è più consona ai nostri tempi. Oggi, la conoscenza e, soprattutto, l'accesso alla conoscenza sono più facili, più diffusi. La presenza di protocolli e di Linee Guida indirizza meglio l'attività del medico e, soprattutto, la complessità della medicina e la sua specializzazione hanno fatto sì che, in alcuni settori e in alcune particolarità, molti medici, fortunatamente, hanno competenze ed esperienze ben maggiori rispetto al "Primario".

E, allora, il ruolo del Direttore di struttura complessa va ridefinito, almeno nelle nostre realtà. Dobbiamo chiederci, infatti, quali caratteristiche devono essere richieste e quali competenze cliniche, organizzative e motivazionali bisogna avere per aspirare a ricoprire questa carica.

Inizierei da quelle scientifiche, che possiamo graduare, ma non troppo, sulla base della complessità e dell'importanza della struttura da dirigere.

E, quindi, bisogna stabilire come va valutato il merito.

Non certo e non solamente sulla base degli eventuali incarichi alla persona, conseguiti nel tempo, cioè in base alla fiducia. Vorrei dire che c'è un solo metodo, ma so che molti avrebbero da ridire, abituati a valutazioni professionali fatte per sentito dire, ed è quello della valutazione dei risultati clinici conseguiti. Casistiche dei ricoveri, tassi di mortalità su malattie croniche, curve di sopravvivenza, modelli organizzativi realizzati, interventi chirurgici effettuati come primo operatore, tipologia e grado di difficoltà degli stessi e valutazione dei re-interventi effettuati, della sopravvivenza ottenuta o della mortalità verificatasi. 
E, poi, bisogna sapere in quali campi della conoscenza medica ha conseguito importanti risultati. Su quali patologie il soggetto in questione ha effettuato raccolte sistematiche dei dati e quante pubblicazioni su riviste internazionali con un certo Impact Factor ha al suo attivo. Quante citazioni su PubMed sono presenti a suo nome $(0,5,10,15$ o più). $E$, ancora, in quanti congressi nazionali e/o internazionali è stato relatore e su quali tematiche, a quali società scientifiche è iscritto, a quali riviste scientifiche è abbonato e quali strumenti moderni della conoscenza scientifica sa usare.

La valutazione del merito serve per selezionare i Direttori di struttura complessa che realizzino gli obiettivi generali delineati. Ecco perché dovremmo vedere con preoccupazione il fenomeno dei Direttori di struttura complessa incaricati. Nel nostro servizio sanitario ce ne sono troppi. L'incarico, come tutti sanno, si basa sull'anzianità di servizio e spesso viene dato a medici alla fine della loro carriera, che non hanno più ambizioni, voglia di innovare. Ma troppe strutture non possono restare anni in questo limbo. La scelta di un Direttore di struttura complessa va preparata nel tempo; è un compito importante del Direttore generale, che deve poter scegliere fra una lista di medici molto preparati e deve fare in modo che il reparto non si privi di chi, alla fine, non avrà vinto la selezione. In ospedale e nelle ASL deve esserci sempre spazio per i bravi medici.

So bene che i dispositivi che regolano la selezione dei Direttori di struttura complessa sono ben delineati per legge, ma ciò non toglie che, oltre alla valutazione scientifica delineata sopra, sia necessario chiedere ai candidati qualcosa in più. Una valutazione ponderata della situazione clinico-organizzativa della struttura che si andrà a dirigere con indicazioni e suggerimenti sugli obiettivi che il candidato vorrà conseguire. Volume di attività da conseguire, degenza media da ottenere, valorizzazione delle qualità di ciascun medico, crescita professionale di medici e infermieri e impegno, affinché la struttura possa raggiungere gli obiettivi previsti. Questo è il punto centrale, sempre disatteso e che, alla fine, impedisce la possibilità di una valutazione del merito nel tempo del Direttore di struttura complessa e lo costringe a estenuanti trattative con la Direzione dell'ospedale e della ASL. Pertanto, è necessario garantire che gli strumenti indispensabili per conseguire i risultati clinici e organizzativi che saranno inseriti nel contratto fra la Direzione generale dell'ospedale o della ASL e il Direttore di struttura complessa siano e rimangano certi. Se noi vogliamo fare una valutazione dei risultati conseguiti e del rinnovo o meno del contratto, dobbiamo garantire tutti i mezzi necessari per il loro conseguimento. Lo accenno per precisarlo magari meglio parlando dei Direttori generali. Ammettiamo di stabilire che, in un reparto con 20 posti letto, debbano essere presenti 4 infermieri la mattina, 4 la sera e 2 la notte. Se una mattina ne manca 1 perché ha la febbre, cosa facciamo? Direi che i restanti infermieri cercheranno di sopperire alla mancanza del loro collega lavorando di più e garantendo la normale attività del reparto. $\mathrm{Ma}$, se una mattina sono in 2 a essere assenti per malattia, cosa facciamo? Deve esistere un meccanismo automatico e collaudato per cui, da qualche parte, dall'interno dell'ospedale o dall'esterno (una società che fornisce infermieri certificati idonei al lavoro richiesto), almeno 1 infermiere venga sostituito in tempi rapidi. Come risulta evidente, se mancano due infermieri non solo l'attività assistenziale sarà ridotta, ma tutte le attività di diagnosi e cura saranno allungate, con un aumento della durata della degenza, dei costi e delle sofferenze.

In questo modo, migliorando questi aspetti organizzativi, potremo chiedere e avere la certezza che gli obiettivi stabiliti verranno raggiunti.

\section{Riassunto}

In questi anni ci troviamo di fronte a cambiamenti a cui non eravamo preparati, anche se, altrove, questi cambiamenti erano già stati affrontati. I costi sanitari stanno diventando progressivamente più alti e, per certi versi, insostenibili perché i cambiamenti demografici stanno portando a un progressivo invecchiamento della popolazione a causa di bassi tassi di natalità e dell'aspettativa di vita più lunga. Sempre più, però, la vita più lunga è vissuta in solitudine. A più risorse investite, quindi, non corrispondono i risultati di salute desiderati.

L'invecchiamento della popolazione, il benessere che ha cancellato molte malattie e uno stile di vita sedentario che ne ha portato di nuove hanno aumentato sia le malattie degenerative che quelle cardiovascolari insieme ai tumori, con il collante di obesità e diabete.

Per cui, più organi si ammalano, contemporaneamente, per gli stessi motivi e per gli stessi fattori di rischio.

Quindi, bisogna iniziare ad aggregare culture mediche frammentate e a mettere l'uomo al centro delle cure.

Dobbiamo riconoscere, dunque, che i modelli organizzativi devono adattarsi ai cambiamenti epidemiologici e che questi cambiamenti coinvolgono le professioni, medici e infermieri, cambiandone il ruolo, i poteri e le responsabilità e il modo di lavorare. Essi non dovranno più occuparsi solamente della cura degli organi ammalati, come hanno sempre fatto, ma sempre più dovranno prendersi cura di pazienti ammalati a più organi.

Parole chiave: Modelli organizzativi, Competenze mediche, Valutazioni, Progressione di carriera

Dichiarazione di conflitto di interesse: L'Autore dichiara di non avere conflitto di interessi.

Contributi economici agli Autori: L'Autore dichiara di non avere ricevuto sponsorizzazioni economiche per la preparazione dell'articolo.

\section{Indirizzo dell'Autore:}

Dr. Antonio Barracca

Medico, Specialista in Nefrologia e Urologia

Viale Merello 74

09123 Cagliari

barraccaantonio@gmail.com 